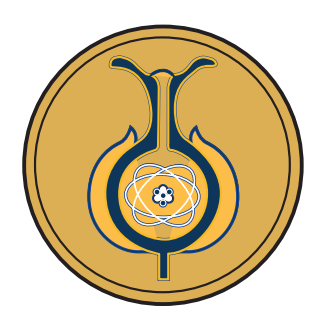

\title{
Medición de la huella hídrica azul de la Universidad Nacional en Costa Rica, del 2012 al 2016
}

\author{
Measurement of blue water footprint in Universidad Nacional in Costa Rica \\ from 2012 to 2016
}

Medição da pegada hídrica azul da Universidade Nacional da Costa Rica, de 2012 a 2016

Fabián Chavarría-Solera

fchavar@una.ac.cr

Universidad Nacional,

UNA-Campus Sostenible,

Heredia, Costa Rica.

Orcid: https://orcid.org/0000-0001-9111-4860

Jeison Rodríguez-Flores

jeison200916@hotmail.com

Universidad Nacional,

UNA-Campus Sostenible,

Heredia, Costa Rica.

Orcid: https://orcid.org/0000-0002-8151-7751

Andrea Herrera-Araya

andre.herrera.araya@gmail.com

Universidad Nacional,

UNA-Campus Sostenible,

Heredia, Costa Rica.

Orcid: https://orcid.org/0000-0002-9808-6935
Rebeca Gamboa-Venegas

rebe.gamb07@gmail.com

Universidad Nacional,

UNA-Campus Sostenible,

Heredia, Costa Rica.

Orcid: https://orcid.org/0000-0001-5354-7470

\section{Diana Chinchilla-González \\ ddianacg95@gmail.com \\ Universidad Nacional, \\ UNA-Campus Sostenible, \\ Heredia, Costa Rica.}

Orcid: https://orcid.org/0000-0001-5427-2317

Ana Carolina Herra-Solís

carolis ch@hotmail.com

Universidad Nacional,

UNA-Campus Sostenible,

Heredia, Costa Rica.

Orcid: https://orcid.org/0000-0002-5354-3303

Received: 21/Jan/2019 • Accepted: 27/Jul/2019 • Published: 31/Jan/2020

\section{Resumen}

La cuantificación y medición de la huella hídrica permite analizar y tener un control sobre la gestión ambiental de la institución, además de tomar acciones que integren la totalidad de la población universitaria, orientando todos los esfuerzos hacia un desarrollo sustentable. El objetivo de la investigación es cuantificar y calcular la huella hídrica azul institucional para el periodo 2012-2016, con el fin de analizar y comparar, así, su comportamiento en el tiempo, de acuerdo con las variables contempladas en el cálculo, consumo de agua, consumo de combustible fósil, papel y energía eléctrica (puesto que se consideran fundamentales para el desenvolvimiento y funcionalidad de actividades institucionales). Se aplicó la metodología de Hoekstra y Chapagain, expuesta en el Manual de evaluación de la huella hídrica; con los factores de huella hídrica y agua virtual, tomados de Water Footprint Network, se obtuvo la cantidad correspondiente a cada rubro. Como resultado, entre el 2012 y el 2015, se llegó a una disminución del 8 \% de la huella hídrica, pasando de $966432 \mathrm{~m}^{3}$ a $888693 \mathrm{~m}^{3}$. Mientras tanto, en el 2016, se alcanzó un leve aumento, dando un valor total de $891976 \mathrm{~m}^{3}$. 
Palabras clave: Agua virtual; indicador ambiental; consumo de agua; huella hídrica azul.

\section{Abstract}

Quantifying and measuring the water footprint allows for the analysis and control over the environmental management of the institution and helps integrate college population, thus directing all efforts towards a sustainable development. The objective of this research is to quantify and calculate the institutional blue water footprint for the 2012-2016 period to analyze and compare its behavior over time, according to the variables included in the calculation such as consumption of water, fossil fuel, reams of paper and electrical energy (since they are important for the function and performance of institutional activities). The methodology applied was the one described by Hoekstra and Chapagain in the Water Footprint Assessment Manual, as well as the water footprint and virtual water factors taken from the Water Footprint Network. The corresponding amount was obtained for each item. As a result, between 2012 and 2015, the Institutional Water Footprint decreased 8\%, from $966432 \mathrm{~m}^{3}$ to $888693 \mathrm{~m}^{3}$, while in 2016 it slightly increased to $891976 \mathrm{~m}^{3}$.

Keywords: virtual water; environmental indicator; water consumption; blue water footprint.

\section{Resumo}

A quantificação e mensuração da pegada hídrica permite analisar e controlar a gestão ambiental da instituição, além de realizar ações que integram toda a população universitária, orientando todos os esforços em direção ao desenvolvimento sustentável. 0 objetivo da pesquisa é quantificar e calcular a pegada hídrica azul institucional entre 2012 e 2016, a fim de analisar e comparar, assim, seu comportamento ao longo do tempo, de acordo com as variáveis contempladas no cálculo, como o consumo de água , 0 consumo de combustíveis fósseis, resmas de papel e energia eléctrica (por serem considerados essenciais para o desenvolvimento e a funcionalidade das atividades institucionais). A metodologia de Hoekstra e Chapagain, estabelecida no Manual de Avaliação da Pegada Hídrica, foi aplicada e, com os fatores de pegada hídrica e água virtual, obtidos da Water Footprint Network, foi obtido o valor correspondente a cada item. Como resultado, entre 2012 e 2015, foi alcançada uma redução de 8\% na pegada hídrica, de 966432 $\mathrm{m}^{3}$ para $888693 \mathrm{~m}^{3}$. Enquanto isso, em 2016, foi alcançado um ligeiro aumento, totalizando $891976 \mathrm{~m}^{3}$.

Palavras-chaves: água virtual; indicador ambiental; consumo de água; pegada hídrica azul.

\section{Introducción}

$\mathrm{E}$ n la II Cumbre sobre Desarrollo Sustentable de Johannesburgo, en 2001, en que se trató la escasez de agua potable y sus consecuencias, se señalaron cifras alarmantes: un quinto de la población mundial no tiene acceso a dicho recurso (2.400 millones de personas), situación que tiene todos los perfiles de una explosiva bomba de tiempo que la humanidad ha comenzado a vivir en su tercer milenio. En el Foro Mundial del Agua, desarrollado en
La Haya Holanda, se insistió en un contrato mundial del agua, que partiera de un conjunto de principios básicos, entre estos que el agua es fuente de vida y como tal es un bien común que no pertenece a los países, sino al conjunto de la sociedad planetaria; el acceso al agua es un derecho no sujeto a discusión alguna y la responsabilidad del acceso es colectiva (Agudelo, 2005).

En los últimos siglos, la población mundial ha crecido de manera acelerada, y los recursos que antes se concebían como inagotables comenzaron a escasear 
en algunos sectores del planeta. El principal problema de la escasez se nota en las cantidades de agua potable que están disponibles para el consumo; solamente una pequeña porción del total de agua en el planeta es agua potable (aprox. un 2,5\% del total) (Fundación MAPFRE, 2011). Por ello, es indispensable crear una conciencia a nivel mundial de la cantidad que se aprovecha de este bien y buscar alternativas para hacer un mejor uso del recurso.

El agua a nivel institucional se utiliza en el consumo para beber, riego, retretes, lavado de vehículos, limpieza en general, duchas y grifos para el aseo personal y lavado de manos, servicios de alimentación, laboratorios, así como el llenado de piscinas para recreación o deporte. Para abastecerse y realizar estas actividades, la Universidad Nacional (UNA) para el año 2016 reporta un consumo total de 182005 metros cúbicos al año, lo que equivale a 18 litros de agua al día por persona o 6,57 metros cúbicos de agua por año (Chavarría, 2019). Otras instituciones como la Universidad de Córdoba Campus Montería, para el año 2014 reporta un consumo de 164 963,3 metros cúbicos al año (Contreras \& Torres, 2016). La Universidad Católica del Perú campus de la Pontificia, según datos de Castillo (2016) tiene un consumo total anual de 405021 metros cúbicos para el año 2014 y un consumo per cápita de 11,27 metros cúbicos por año.

A través de los años, las comunidades educativas han manifestado su atención por disminuir el volumen de agua utilizado en las labores cotidianas, por lo que es muy importante que las instituciones lleven un registro y control de los consumos de agua como un indicador ambiental, para saber si los cambios de tecnologías, estrategias de ahorro o campaña de concientización con participación activa tanto de personal como de estudiantes tienen efectos positivos sobre el uso eficiente y ahorro para disminuir el consumo de este recurso natural tan valioso.

En nuestro país, según los estudios de la Water Footprint Network (2016), el consumo de agua per cápita es de 1490 metros cúbicos de agua por año, el cual está por encima del promedio mundial, que según indica es de 1385 metros cúbicos por año, esto nos muestra que en Costa Rica se hace un uso mayor del recurso hídrico, en muchas actividades diferentes que pueden ir desde actividades sumamente esenciales; como lo es la preparación de alimentos o el aseo personal, hasta acciones que no necesariamente requieren el uso de agua potable como el lavado de autos, el riego del jardín, entre otras.

Sin embargo, se debe medir y contabilizar el consumo de este recurso no solo de forma directa, sino también indirecta, para tener un mejor y mayor control de este, como lo es el caso del indicador ambiental de huella hídrica. El concepto de huella hídrica $(\mathrm{HH})$ fue definido por primera vez por Hoekstra (2003) como un indicador de desarrollo que se utiliza para cuantificar la cantidad de agua que se consume o que se contamina en la creación de un bien o servicio determinado, con el fin de conocer los impactos y luego poder sugerir políticas de mitigación o cambio en el método productivo. Este autor la define como el volumen total de agua dulce utilizada para producir los bienes y servicios consumidos por el individuo, empresa o nación (Hoekstra et al., 2011).

En ese sentido, la huella hídrica es un indicador de toda el agua que se utiliza en la vida diaria para producir la comida, en procesos industriales y generación de energía, así como la que se ensucia y contamina a través de esos mismos procesos, por ende, su cálculo se puede realizar 
a diferentes niveles de agregación y su ejecución varía, ya sea a nivel nacional, empresarial / institucional o personal (Hoekstra, 2003). La huella de un individuo, por ejemplo, se expresa en términos de volumen, consumido por año, de agua fresca; la de un producto se refiere al volumen de agua fresca, utilizado por unidad de producto, ya sea aquella que se ha evaporado o contaminado y que ya no está disponible en su estado original (Hoekstra, 2008).

$\mathrm{Su}$ cálculo debe realizarse de manera directa e indirecta, por medio del concepto de agua virtual que se define más adelante. Por tanto, el concepto de huella hídrica fue introducido con el fin de tener un indicador de consumo basado en el uso del agua; este podría aportar información útil, además de los indicadores tradicionales basados en el uso de agua por sector (Van Oel \& Hoekstra, 2010).

En síntesis, la huella hídrica se considera un indicador global y multidimensional, que permite medir la apropiación de recursos de agua dulce, superando la medición tradicional y limitada de extracción de agua. Según la Water Footprint Network (2016), ofrece una perspectiva mejor y más amplia sobre cómo un sujeto consumidor o productor afecta el uso de sistemas de agua dulce, a su vez que indica dónde, cuándo y cuánta agua se consume y contamina, considerando todos los procesos de la cadena productiva, a través de la clasificación de huella hídrica directa e indirecta. La primera hace referencia al volumen de agua incorporado al producto en las fases operativas; mientras que la segunda corresponde a la que se utilizó en la cadena de suministros, por ejemplo, en la materia prima que sirve de insumo para elaborar el producto (Hoekstra et al., 2011b).

Un estudio de huella hídrica puede emprenderse por muchas razones diferentes. Por ejemplo, una nación puede estar interesada en conocer su dependencia de recursos hídricos extranjeros o puede estar interesada en saber la sostenibilidad del uso del agua en las áreas donde los productos importados hacen uso intensivo del agua en su origen. Una institución puede estar interesada en saber su dependencia sobre los escasos recursos hídricos en su cadena de suministros o cómo puede contribuir a disminuir más los impactos sobre los sistemas de agua durante toda su cadena de suministros y dentro de sus propias operaciones (Loaiza \& Quiceno, 2018). Por su parte, en una institución de educación superior puede ser utilizada para medir el volumen total de agua dulce consumida de forma directa o indirecta por la población en un determinado tiempo. Las instituciones se han dado a la tarea de buscar no solo alternativas físicas, sino también metodologías que enfoquen el gasto inapropiado del recurso en un proceso habitual. Al respecto, el cálculo de huella hídrica resulta una alternativa eficiente para lograr dichos objetivos.

La huella hídrica es un indicador compuesto por tres variables, que se definen de la siguiente manera (Hoekstra \& Chapagain, 2004):

- Huella hídrica azul se refiere al consumo de los recursos hídricos azules (agua dulce), superficial o subterránea, a lo largo de toda la cadena de producción de un producto; el consumo se refiere a la pérdida de agua en cuerpos de agua disponibles en la superficie o en acuíferos subterráneas. La pérdida ocurre cuando el agua se evapora, regresa a otra zona de captación o al mar o se incorpora a un producto. Es decir, la huella hídrica azul mide la cantidad de agua disponible en un determinado período, la que se 
consume sin devolverse a la misma cuenca hídrica. Se excluye de esta medición el volumen de agua azul que retorna a la fuente hídrica tanto luego del uso o por filtrado antes del uso.

- Huella hídrica verde se refiere al consumo de los recursos de agua de lluvia, en la medida en que no se pierde por filtro o río abajo. Es el agua que alimenta el sistema como lluvia y acumula humedad en el suelo, es absorbida por plantas y luego exhalada por flujo de vapor. La huella hídrica verde es el volumen de agua de lluvia consumida durante un proceso de producción, relevante sobre todo para los productos agrícolas y forestales; sin embargo, los productos industriales cuya materia prima es madera o productos a base de cultivo también tienen una huella de agua verde de manera indirecta.

- Huella hídrica gris se refiere a las cantidades de agua requeridas para que esta pueda asimilar cargas de contaminantes en el ámbito de estándares establecidos por normativas ambientales vigentes.

La fórmula general se expresa como:

$$
\mathrm{HH}=\mathrm{HHazul}+\mathrm{HHverde}+\text { HHgris }
$$

La fórmula anterior indica que la huella hídrica se compone de la sumatoria de la huella azul, la huella verde y la huella gris. Es necesario considerar que la evaluación de la huella hídrica se puede realizar a todo tipo de actividades: sea un proceso, producto, ente productor o consumidor, así como a un espacio y tiempo de una zona geográfica específica.
Otro punto indispensable para tomar en cuenta es el cálculo de consumo indirecto de recurso hídrico que se realiza a la hora de consumir un bien; esta se conoce como agua virtual, concepto paralelo al de huella hídrica indirecta. Inicialmente este concepto fue propuesto por John Anthony Allan en el año 1993, quien lo define como el total de agua requerida para la fabricación de un producto, tomando en consideración la cantidad real utilizada en todos los procesos anteriores (Allan, 1993). En otras palabras, es la cantidad de agua contenida en un producto.

Por lo anterior, la huella hídrica es la sumatoria del agua virtual de los distintos productos o procesos más la huella hídrica directa. Se debe considerar el Agua virtual del proceso de distribución. Se relaciona el consumo de energía del transporte con el consumo de agua. Agua virtual de la energía: cantidad de agua que ha hecho falta para producir energía consumida por el transporte.

Huella hídrica $=\mathrm{HH}_{\text {ConsumoDirecto }}+\mathrm{AV}_{\text {ConsumoIndirecto }}$ HH: Huella hídrica AV: Agua virtual

A la hora de cuantificar este indicador existe una diferencia que depende del nivel al cual se desea realizar el estudio, ya que es diferente su ejecución si es nacional, empresarial/institucional o personal (Gerbens-Leenes et al., 2009). En este caso, el cálculo que debe realizarse es el de la huella hídrica a nivel institucional, se revisará el consumo total y per cápita del recurso hídrico en metros cúbicos del personal y estudiantes de la UNA. De manera más simplificada, se obtiene que la huella hídrica de un ente consumidor, o en este caso de una institución, corresponde a la inclusión de los conceptos de huella directa e indirecta. La primera es el consumo de agua personal por parte de la instancia y la segunda refiere 
al agua necesaria para la producción de bienes y servicios utilizados por la instancia en estudio. El uso indirecto del agua se calcula multiplicando todos los productos consumidos por su respectiva huella hídrica del producto (Hoekstra et al., 2011).

Es importante mencionar que, además de la huella hídrica, las instituciones tienen otras maneras de medir la cantidad de agua consumida dentro de sus organizaciones. En nuestro país destaca el Programa de Bandera Azul Ecológica y el Programa de Gestión Ambiental Institucional, los cuales miden la utilización de los recursos institucionales, establecen metas y parámetros de cumplimiento $\mathrm{y}$, a su vez, permiten establecer estrategias enfocadas a la adecuada gestión ambiental. A diferencia de la medición de la huella hídrica, estos programas no toman en consideración la utilización indirecta del recurso hídrico.

Paralelo a esto, existen diferentes certificaciones internacionales relacionadas con el uso de recursos naturales institucionales como la ISO 140001. Derivada de esta certificación, la ISO 14044 mide la huella de agua con base en la metodología del ciclo de vida de un producto. Esta toma en consideración las diferentes huellas de agua dentro de las etapas de producción del producto, identifica cambios en la cantidad de consumo y la calidad del agua y utiliza conocimientos hidrológicos, lo que la hace una metodología bastante completa; pero, a su vez, necesita gran cantidad de insumos y mediciones de campo, lo que eleva los costos de realización.

Este tipo de indicadores ambientales se ha ido desarrollando hasta alcanzar un alto grado de importancia en las políticas de diferentes países, en una lucha continua por integrar la ecología con el sistema productivo. Costa Rica atiende a este llamado con el deseo de alcanzar la neutralidad en la emisión de carbono para el año 2021. Asimismo, se han introducido esfuerzos por cuantificar estos indicadores que muestren el uso de los recursos, como también mediciones del impacto que genera el uso de algunos insumos en la atmósfera, como la emisión de dióxido de carbono. Entre estos están: la huella ecológica, la huella de carbono y la huella hídrica.

Esta última es el punto de estudio de la presente investigación. El auge en la importancia de la huella hídrica a nivel mundial, y en particular para la UNA, se debe a que da a conocer un dato de lo que realmente se consume en un tiempo determinado, tanto de forma directa como indirectamente a nivel institucional. Específicamente, se mide la huella hídrica de toda la Universidad, para así, con ello, informar mediante esta investigación que no solamente se debe contabilizar el consumo directo, sino también se debe tener presente que, en cada proceso productivo realizado, se va a utilizar una cierta cantidad del recurso con el fin de crear el bien de consumo. Se pretende, así, generar conciencia en el manejo de uno de los bienes más necesario para la vida: el agua.

\section{Metodología}

Se realiza una adaptación de la metodología utilizada por Hoekstra y Chapagain (2011), se llevó a cabo el cálculo de la huella hídrica institucional de la UNA únicamente en el concepto de huella azul, debido a que se tienen los datos necesarios para la cuantificación de sus principales componentes, a su vez, porque es la causante del mayor impacto en la huella hídrica.

El cálculo de la huella hídrica verde se ha utilizado a través de los años para cuantificar el impacto de actividades como la agropecuaria, que necesitan del agua de lluvia para 
su efectiva producción. Por lo tanto, debido a la naturaleza de la actividad principal de la institución, se excluye del cálculo la huella hídrica verde dentro de UNA.

Así mismo, la huella hídrica gris no se toma en consideración en el cálculo por el faltante de datos para su medición, expuesta esta como la cantidad de agua dulce necesaria para recibir el agua contaminada creada por el proceso de consumo o producción.

En el estudio se cuantifico la huella azul de toda la institución, se contemplaron todos los campus universitarios: Campus Omar Dengo, Campus Benjamín Núñez, Campus Coto, Campus Pérez Zeledón, Campus Liberia y Nicoya, Sección Sarapiquí, las cuatro estaciones (ECMAR -Estación Nacional de Ciencias Marino Costeras-, EBM -Estación de Biología Marina-, Estación 28 millas, Estación Río Macho) y Finca experimental Santa Lucía, de la Escuela de Ciencias Agrarias.

Los datos fueron suministrados por las diferentes entidades tanto internas (Proveeduría, Programa de Gestión Financiero (PGF) y Sección de Transporte Institucional) como externas (empresas que brindan los servicios públicos como el suministro de agua potable y energía eléctrica; Empresa de Servicios Públicos de Heredia (ESPH), Instituto Costarricense de Electricidad (ICE), Compañía de Fuerza y Luz (CNFL), Coopeguanacaste y Acueductos y Alcantarillados (AyA). De esta manera se efectúo el cálculo total de la huella hídrica azul institucional y sus diferentes campus, para lo cual se consideraron las variables de consumo de agua, consumo de combustible fósil, utilización de resmas de papel y la energía eléctrica consumida, debido a que cumplen un papel importante en el desempeño de la ejecución de las actividades institucionales. Para obtener la cantidad de población universitaria, se consultaron los datos de matrícula del Departamento de Registro, la planilla laboral del Programa de Desarrollo de Recursos Humanos, así como un documento de población flotante no regular suministrado por el Área de Planificación Económica (APEUNA). No se toma en cuenta la alimentación, pues la universidad no es compradora directa de los productos que se venden en los servicios de alimentación.

En la clasificación de las variables se obtuvieron:

Consumo directo:

- Cantidad de metros cúbicos consumidos.

Consumo indirecto:

- $\quad$ Agua virtual del uso de transporte universitario (combustible fósil).

- $\quad$ Agua virtual del uso de energía eléctrica.

- $\quad$ Agua virtual del uso de papel.

Utilizando la metodología de Hoekstra y Chapagain (2011), expuesta en el Manual de evaluación de la huella hídrica y con los factores de huella hídrica y agua virtual tomados de diferentes estudios de Water Footprint Network (2016), se obtuvo la cantidad correspondiente a cada rubro (Tabla 1 ).

Tabla 1.

Factores de huella hídrica azul contemplados en el estudio

\begin{tabular}{ll}
\hline \multicolumn{1}{c}{ Aspecto ambiental } & \multicolumn{1}{c}{ Factor } \\
\hline Combustible fósil & $0.05 \mathrm{~m}^{3} / \mathrm{L}$ de combustible \\
Papel/Resmas & $5 \mathrm{~m}^{3} / /$ Resma \\
Energía eléctrica* & $22 \mathrm{~m}^{3} / 277,77 \mathrm{~kW} / \mathrm{h}$ \\
Consumo de agua & $\mathrm{m}^{3}$
\end{tabular}

Nota: Fuente propia de la investigación con datos de Water Footprint Network (2016).

*En el caso de la energía eléctrica, la mayoría de la producción es hidroeléctrica, por lo que el factor de cálculo corresponde a $22 \mathrm{~m}^{3} / \mathrm{kW} / \mathrm{h}$. 
Se aplicó la siguiente fórmula:

$$
\mathrm{HH}_{\text {UNA/AÑO }}=\mathrm{HH}_{\text {Consumodirecto/Año }}+\mathrm{AV}_{\text {Consumoindirecto/Año }}
$$

Dónde:

$$
\mathrm{AV}_{\text {Consumoindirecto Año }}=\sum \mathrm{C}(\mathrm{p}) \times \mathrm{HH}(\mathrm{p})
$$

En el que:

$\mathrm{C}(\mathrm{p})$ : consumo anual del producto o servicio.

HH(p): Huella hídrica de cada producto o servicio, en este caso del papel, combustible y energía eléctrica, medida como la cantidad de agua utilizada para la creación de una unidad de producto.

Para obtener el resultado de huella hídrica per cápita al año, de los aspectos de energía eléctrica y agua se dividió el resultado de metros cúbicos referente a cada uno al año entre la cantidad de población universitaria (personal y estudiantes). Por su parte, la huella hídrica per cápita al año

\section{Gráfica 1}

Total de huella hídrica azul institucional de la UNA para el periodo 2012-2016

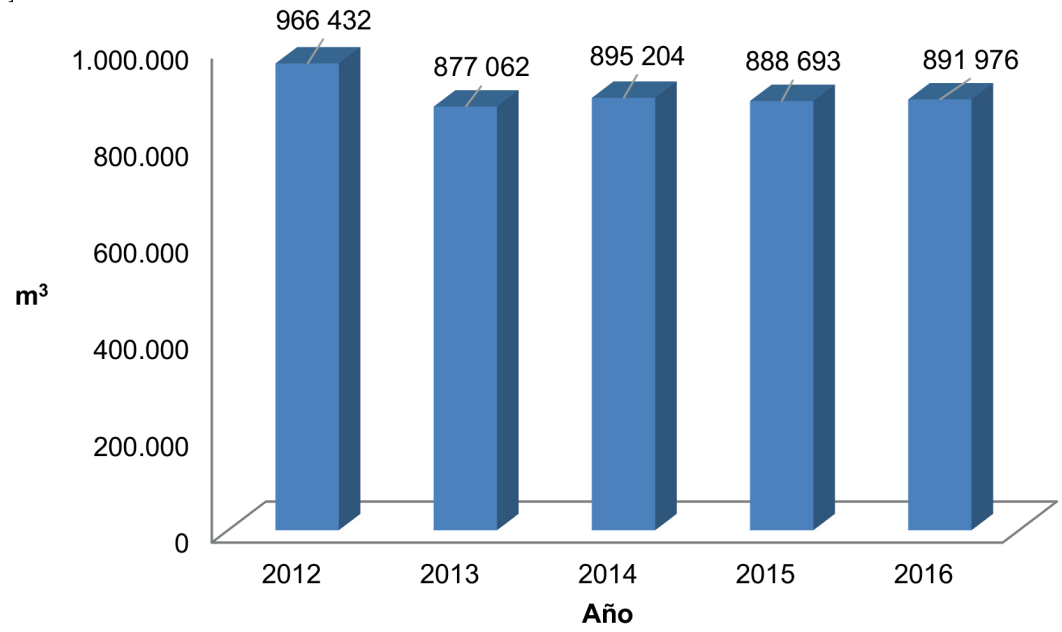

Nota: Fuente propia del estudio con datos del Plan del Programa de Gestión Ambiental Institucional de la Universidad Nacional de Costa Rica PGAIUNA 2017-2021 (2017). concerniente a los aspectos de combustible fósil y papel se calculó dividendo el resultado de metros cúbicos referente cada uno al año entre la cantidad de personal. Lo anterior se debe a que el combustible de la flotilla vehicular y las resmas de papel solo son consumidos por funcionarios y funcionarias, y no por estudiantes.

\section{Resultados y discusión}

Para obtener el resultado de la huella hídrica per cápita total al año, se procedió a sumar la huella hídrica resultante de cada uno de los aspectos considerados en el cálculo.

En la gráfica 1, se visualiza el comportamiento en metros cúbicos de la huella hídrica azul total de toda la institución desde el año 2012 hasta el 2016. Esta información muestra, de manera general, que se ha presentado un comportamiento de disminución del ocho por ciento en el indicador ambiental durante el periodo comprendido. Para el año 2012 el resultado de este fue de 966432 $\mathrm{m}^{3}$, en el 2013 este resultado presentó una baja del nueve por ciento (9 $\%$ ); en valores reales es de $877062 \mathrm{~m}^{3}$ cifra que corresponde al resultado más bajo durante el periodo de estudio. Para el 2014 y 2015 , los resultados fueron de $895204 \mathrm{~m}^{3}$ y $888693 \mathrm{~m}^{3}$ respectivamente, generándose una leve disminución del uno por ciento $(1 \%)$, igual reducción $(8 \%)$ se presentó para el año 2016 con un valor total de $891976 \mathrm{~m}^{3}$ (Gráfico 1). 
En la Tabla 2 se muestran los resultados generales sobre todos los aspectos ambientales analizados en el estudio. Acá se presentan los diferentes componentes utilizados en la medición del indicador para los cinco años en análisis y se incluyen, entre otros: la cantidad correspondiente y el porcentaje de participación de cada aspecto ambiental en la medición.
El consumo de energía eléctrica de la UNA ha experimentado un comportamiento de incremento desde el año 2012. En ese año fue de $6818915 \mathrm{KW} / \mathrm{h}$ y ascendió hasta los $7624841 \mathrm{KW} / \mathrm{h}$ en 2015, representando un aumento del doce por ciento $(12 \%)$ en el periodo comprendido. El incremento en el consumo se explica por motivos de crecimiento de la población universitaria en 33

Tabla 2

Huella hídrica azul de la Universidad Nacional para el periodo 2012-2016

\begin{tabular}{|c|c|c|c|c|c|c|c|}
\hline \multirow{2}{*}{$\begin{array}{c}\text { Aspecto } \\
\text { ambiental }\end{array}$} & \multirow[t]{2}{*}{ Indicador } & \multicolumn{5}{|c|}{ Año } & \multirow[t]{2}{*}{ Totales } \\
\hline & & 2012 & 2013 & 2014 & 2015 & 2016 & \\
\hline \multirow[t]{4}{*}{$\begin{array}{l}\text { Energía } \\
\text { eléctrica }\end{array}$} & $\begin{array}{l}\text { Cantidad } \\
(\mathrm{KW} / \mathrm{h})\end{array}$ & 6818915 & 6594219 & 7472720 & 7624841 & 7915637 & 36426332 \\
\hline & $\begin{array}{l}\text { Huella hídrica Azul } \\
\left.\text { institucional ( } \mathrm{m}^{3} / \mathrm{anno}\right)\end{array}$ & 540073 & 522277 & 591856 & 603904 & 626936 & 2885046 \\
\hline & $\begin{array}{l}\text { HH Per cápita }\left(\mathrm{m}^{3} /\right. \\
\text { año/persona) }\end{array}$ & 26 & 24 & 24 & 22 & 23 & 119 \\
\hline & $\mathrm{HH}(\%)$ & 56 & 60 & 66 & 68 & 70 & - \\
\hline \multirow[t]{4}{*}{ Agua } & Cantidad $\left(\mathrm{m}^{3}\right)$ & 329262 & 261095 & 226638 & 203015 & 182005 & 1202015 \\
\hline & $\begin{array}{l}\text { Huella hídrica Azul } \\
\text { institucional ( } \mathrm{m}^{3} / \mathrm{año} \text { ) }\end{array}$ & 329262 & 261095 & 226638 & 203015 & 182005 & 1202015 \\
\hline & $\begin{array}{l}\text { HH Per cápita }\left(\mathrm{m}^{3} /\right. \\
\text { año/persona })\end{array}$ & 16 & 12 & 9 & 7 & 7 & 51 \\
\hline & $\mathrm{HH}(\%)$ & 34 & 30 & 25 & 23 & 20 & - \\
\hline \multirow{4}{*}{$\begin{array}{l}\text { Combustible } \\
\text { fósil }\end{array}$} & Cantidad (L) & 317936 & 310906 & 311603 & 332080 & 329803 & 1602328 \\
\hline & $\begin{array}{l}\text { Huella hídrica Azul } \\
\text { institucional }\left(\mathrm{m}^{3} / \mathrm{año} o\right)\end{array}$ & 15897 & 15545 & 15580 & 16604 & 16490 & 80116 \\
\hline & $\begin{array}{l}\text { HH Per cápita }\left(\mathrm{m}^{3} /\right. \\
\text { año/persona) }\end{array}$ & 4 & 4 & 4 & 5 & 4 & 22 \\
\hline & $\mathrm{HH}(\%)$ & 2 & 2 & 2 & 2 & 2 & - \\
\hline \multirow[t]{4}{*}{ Papel } & Cantidad (Resmas) & 16240 & 15629 & 12226 & 13034 & 13309 & 70438 \\
\hline & $\begin{array}{l}\text { Huella hídrica Azul } \\
\text { institucional }\left(\mathrm{m}^{3} / \mathrm{año} o\right)\end{array}$ & 81200 & 78145 & 61130 & 65170 & 66545 & 352190 \\
\hline & $\begin{array}{l}\text { HH Per cápita }\left(\mathrm{m}^{3} /\right. \\
\text { año/persona) }\end{array}$ & 23 & 22 & 17 & 19 & 18 & 98 \\
\hline & $\mathrm{HH}(\%)$ & 8 & 9 & 7 & 7 & 7 & - \\
\hline Total & $\begin{array}{l}\text { Huella hídrica Azul } \\
\text { institucional ( } \mathrm{m}^{3} / \text { año) }\end{array}$ & 966432 & 877062 & 895204 & 888693 & 891976 & 4519368 \\
\hline $\begin{array}{l}\text { Total Per } \\
\text { cápita }\end{array}$ & $\begin{array}{l}\text { Huella hídrica Azul } \\
\text { institucional ( } \mathrm{m}^{3} / \mathrm{año} / \\
\text { persona) }\end{array}$ & 69 & 62 & 54 & 53 & 52 & 291 \\
\hline \multicolumn{2}{|c|}{ Población (estudiantes + personal) } & 20683 & 21574 & 24898 & 27517 & 27050 & - \\
\hline \multicolumn{2}{|c|}{ Población (personal) } & 3557 & 3580 & 3549 & 3452 & 3774 & - \\
\hline
\end{tabular}

Nota: Fuente propia de la investigación con datos del Plan del Programa de Gestión Ambiental Institucional de la Universidad Nacional de Costa Rica PGAI-UNA 2017-2021 (2017) y entes proveedores de servicios de energía eléctrica y agua potable. 
$\%$ en el periodo 2012-2015 (UNA, 2017), así como el aumento en los medidores por concepto de alquileres para centros de trabajo y construcción de nuevos edificios. Sin embargo, es importante mencionar que ya se han implementado nuevos dispositivos para el ahorro de la energía eléctrica en varios campus, centros e instancias como, por ejemplo, instalación de luz LED, paneles solares y aires acondicionados SEER 16 de tecnología INVERTER con eficiencia energética alta y de suave arranque, sin picos de corriente (Chavarría et al., 2015).

Por otra parte, en la UNA se ha venido presentando una disminución en los metros cúbicos $\left(\mathrm{m}^{3}\right)$ anuales consumidos de agua por toda la institución. En el año 2012 fue de $329262 \mathrm{~m}^{3}$ /año y disminuyó en un 21 $\%$ en el 2013 para un total de $261095 \mathrm{~m}^{3}$ / año. Durante el 2014 se consumió un total de $226638 \mathrm{~m}^{3}$; en el 2015, un total de 203015 $\mathrm{m}^{3} \mathrm{y}$ en el año 2016, un total de $182005 \mathrm{~m}^{3}$, lo cual representa un ahorro del $45 \%$ durante todo el periodo de estudio (UNA, 2017). Como se observa, hubo un considerable ahorro en la utilización del recurso hídrico, pese al aumento de la población universitaria y al incremento en la cantidad de medidores activados, debido a nuevas infraestructuras, así como a alquileres. Para esta reducción es importante destacar la adquisición e instalación de nuevos dispositivos y tecnologías más eficientes de ahorro de agua, como los mingitorios libres de agua.

Actualmente se reemplazaron mingitorios de agua por un total de 118 unidades libres de agua en el Campus Omar Dengo, según la contratación 2015LA-000117SCA. Anteriormente, ya se habían instalado 27 unidades en varios departamentos de la UNA: Escuela de Ciencias Biológicas, servicio de alimentación de la Facultad de Ciencias Exactas y Naturales, Escuela de
Química, Facultad de Tierra y Mar y en el servicio de alimentación de esta Facultad, Centro de Investigación y Docencia en Educación (CIDE), edificio de la Rectoría, programa Desarrollo y Mantenimiento de Infraestructura Institucional (PRODEMI) y Facultad de Ciencias Sociales. Según indicaciones del ente proveedor, estos nuevos equipos ahorran 151000 litros de agua potable al año (Chavarría et al., 2015). Otras acciones importantes para el éxito en la reducción del gasto de agua son el mantenimiento de las tuberías y tanques de almacenamiento de agua, así como la reparación de fugas.

Las resmas de papel utilizadas en la Universidad han experimentado una reducción de aproximadamente el $18 \%$ durante los últimos cinco años analizados. Esta tendencia es debido a medidas ambientales implementadas con éxito, como lo son la conformación de las comisiones ambientales a nivel de cada facultad, centro y campus regionales encargas de realizar actividades ambientales en pro de la gestión de los residuos aprovechables generados en cada instancia, gestión del consumo de agua, papel, energía eléctrica, combustibles fósiles, entre otros, apoyando de esta forma la implementación del Programa de Gestión Ambiental Institucional. Asimismo, otras medidas que se consideran importantes son la elaboración y ejecución de una oferta de capacitación por parte de UNA-Campus Sostenible, del mismo modo que la continuidad que se le dio a la campaña "Únase al ahorro, cada acción cuenta" donde se incorpora el tema de ahorro de papel 2014-2015, y la iniciativa para establecer lineamientos para la reducción del uso de papel (Chavarría et al., 2015). Además, cabe mencionar que instancias como el Programa de Gestión Financiero han implementado estrategias para el ahorro de papel a lo interno de sus oficinas. 
Actualmente se ha comenzado a utilizar la firma digital en áreas claves de la Universidad como la Proveeduría Institucional y Asesoría Jurídica.

Como resultado, en el año 2012 se consumieron un total de 16240 resmas, que disminuyeron en el 2013 y 2014 en cinco y 22 $\%$ respectivamente. Sin embargo, para el 2015 se da un incremento del siete por ciento: 811 resmas más respecto al 2014. En el 2016 aumenta un dos por ciento (2\%) más y llega a un valor total de 13309 resmas de papel consumidas por el personal de la institución.

Por otra parte, en el consumo de combustible fósil se contemplaron las fuentes móviles, esto es, los automotores, utilizados en giras académicas, de investigación y extensión, así como las fuentes fijas que corresponden a las plantas eléctricas utilizadas para emergencias cuando no hay fluido de energía eléctrica en los edificios. En el 2012 se consumieron 317936 litros, para el 2013 bajo a 310906 litros, lo que representa un ahorro de dos por ciento (2\%). En el año 2013 se

\section{Gráfica 2}

Porcentaje de participación de los aspectos ambientales utilizados en el cálculo de la huella hídrica azul de la Universidad Nacional para el periodo 2012-2016

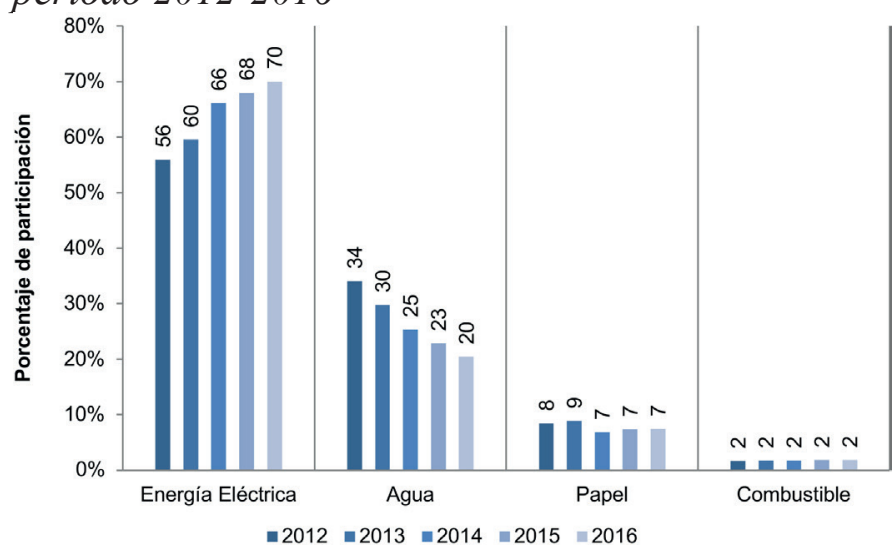

Nota: Fuente propia del estudio con datos del Plan del Programa de Gestión Ambiental Institucional de la Universidad Nacional de Costa Rica PGAI-UNA 2017-2021 (2017). mantiene relativamente constante llegando a un consumo total de 311603 litros representando un aumento de solo 0,2\%. Posteriormente, en el 2014 y 2015 se da un incremento de un siete por ciento en el consumo (7\%), con valores totales de 311603 litros y 332080 litros para cada año. Por último, en el 2016 disminuye en uno por ciento (1\%) y llega a un valor de 329803 litros anuales consumidos por la institución. El consumo de este componente se incrementó en un cuatro por ciento durante el periodo estudiado. Sin embargo, se ha presentado un incremento relativamente bajo, a pesar de que se ha incrementado la flotilla vehicular en un $31 \%$, pues aumentó de 204 a 268 unidades entre el 2012 - 2016, periodo de estudio.

Entre la medidas implementadas en el tema de ahorro de combustible fósil están la sistematización e implementación de un sistema informático con el que se mejoró la eficiencia para la solicitud de giras utilizadas para la investigación y academia, además de la sensibilización y las capacitaciones sobre manejo eficiente para mejorar las buenas prácticas de conducción, mantenimiento correctivo de los vehículos institucionales, compra de vehículos eficientes en el consumo de combustible, así como la adquisición e instalación de un sofisticado sistema de GPS en la mayoría de vehículos de la flotilla institucional, lo que permitió una mejor y adecuada gestión para el ahorro del combustible. Actualmente se le está instalando el sistema GPS al $100 \%$ de la flotilla vehicular (Chavarría et al., 2015).

En el Tabla 2 y el Gráfico 2 se observa que el aspecto ambiental que presentó mayor participación en el cálculo del indicador para todos los años de estudio es el consumo de 
energía eléctrica, representado en todos los años con más del $50 \%$; en el 2012 su participación fue del $56 \%$ y para los siguientes años presenta un aumento promedio de cuatro puntos porcentuales, en el 2015 pasa al $68 \%$ y llega al $70 \%$ en el 2016. Caso contrario al consumo de agua, que muestra una disminución en la participación del cálculo del indicador con el pasar de los años, inicialmente fue del $34 \%$ y al finalizar el estudio solamente representa el $20 \%$, debido a una disminución en los metros cúbicos anuales consumidos por toda la institución.

En lo que respecta al papel y al combustible, el porcentaje de participación de ambos componentes en el cálculo del indicador muestra un comportamiento constante durante el periodo de estudio; no obstante, el en caso específico del papel presenta una variación muy poco significativa de un punto porcentual en los dos primeros años, y un comportamiento constante sin variación alguna para el periodo que resta; a su vez, el consumo de combustible mantiene el porcentaje de participación más bajo y más constante de los cuatro componentes: en todos los años del dos por ciento.

\section{Gráfica 3}

Regresión lineal de la huella hídrica per cápita para los años 2012-2016

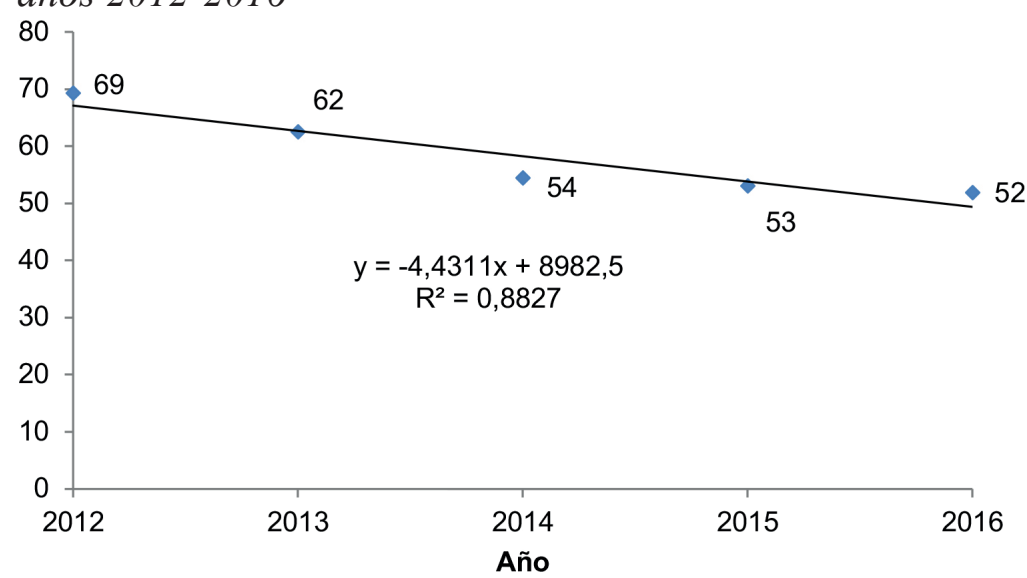

Nota: Fuente propia del estudio con datos del Plan del Programa de Gestión Ambiental Institucional de la Universidad Nacional de Costa Rica PGAI-UNA 2017-2021 (2017).
En cuanto al indicador de huella hídrica per cápita calculado, los datos se presentan en la Tabla 2, en el año 2012 fue de 69 $\mathrm{m}^{3}$ /año/persona descendiendo a $62 \mathrm{~m}^{3} / \mathrm{año} /$ persona en el 2013, posteriormente disminuyendo más en el 2014 a $54 \mathrm{~m}^{3} / \mathrm{año} /$ persona a $53 \mathrm{~m}^{3} /$ año/persona en el 2015 y por último a 52 mªño/persona en el año 2016.

Debido a que se observó una tendencia lineal hacia la disminución en estos datos, se realizó una regresión lineal para obtener la ecuación de la recta $\mathrm{y}=-4,4311 \mathrm{x}+8982,5$ $\left(\mathrm{R}^{2}=0,8827\right)$ (Figura 3). Sustituyendo el año en " $x$ " se pudo predecir que para el 2017 se espera que el agua, al año, que consume una persona en la institución, representado por el cálculo de huella hídrica sea de $45 \mathrm{~m}^{3} / \mathrm{año}$, lo que significaría una disminución del trece por ciento (13\%) con respecto al 2016.

Según Vázquez \& Buenfil (2012), la huella hídrica azul per cápita para Costa Rica es de $110 \mathrm{~m}^{3} / \mathrm{año} /$ persona, con lo que cabe recalcar que el consumo de agua de una persona en la UNA es en promedio la mitad de este valor, no supera los $60 \mathrm{~m}^{3} / \mathrm{año} /$ persona al promediar los cinco años contemplados en el estudio. Los funcionarios y funcionarias están aproximadamente 8 horas presenciales, pero en diferentes turnos que, al menos, son dos turnos; mientras que la población estudiantil está presente en los campus en tiempos reducidos, a excepción de las residencias estudiantiles, donde se presentan hábitos domiciliarios de consumo.

Por su parte, esta tendencia a la reducción que se muestra en el Gráfico 3 es un indicador positivo, el cual demuestra que se están 
teniendo buenos resultados con las acciones implementadas para ahorrar este recurso tan importante para la subsistencia de las presentes y futuras generaciones.

Es importante indicar que otras de las acciones que han venido a consolidar el ahorro de recursos es la concientización a la población universitaria, con el accionar de UNA-Campus Sostenible y del Programa de Gestión Ambiental Institucional, mediante la implementación de medidas ambientales estratégicas en el área educativa, por ejemplo, el lanzamiento y seguimiento a la campaña de ahorro ECO-Oficinas. Esta constituye un compromiso del personal a inscribirse como una oficina comprometida con el ambiente y ser evaluadas periódicamente para ver ese grado de compromiso en el lugar de trabajo, según los aspectos ambientales considerados, además de la utilización de pegatinas informativas y de avisos en partes estratégicas para difundir el uso correcto y ahorrativo de agua y energía. Esta iniciativa, junto con las constantes capacitaciones, tienen como principal objetivo mejorar las prácticas usuales a la hora de utilizar los recursos, generar conciencia en la población universitaria y propiciar un cambio de cultura sobre el correcto uso del recurso hídrico.

La importancia de estudios como este es que demuestran un dato más preciso sobre el consumo del recurso hídrico al utilizar el indicador de huella hídrica y no solo basar los estudios de sostenibilidad ambiental con la cuantificación del consumo de agua directa, ya que la cantidad de agua utilizada va más allá considerando consumos indirectos que inclusive tienen una huella hídrica mayor.

Es importante mencionar que la metodología utilizada según el modelo de cálculo de la Water Footprint Network es fácilmente aplicable y adaptable para instituciones educativas como universidades y puede seguirse usando ampliamente por otras instituciones que requieran medir el consumo de agua y tener una línea base como indicador sobre el cual proponer medidas ambientales correctivas para el ahorro y disminución de este recurso natural.

Sin embargo, aunque la aplicación de este tipo de metodología es relativamente sencilla, se necesita realizar muchos cálculos y hay que tomar en cuenta que una de la principales limitaciones que se presentaron a lo largo de este trabajo de investigación y que se deben considerar para su aplicación en otros casos es la falta de información expedita para el mantenimiento de las bases de datos y cálculos de este indicador, por tanto, es importante llevar un registro ordenado de toda la información de los recursos e insumos necesarios para el funcionamiento de una institución de educación superior.

Para futuras investigaciones se recomienda dar continuidad a este estudio y realizar el cálculo de la huella hídrica de la Universidad tomando en cuenta, además de la huella hídrica azul, la gris y la verde, y también realizarlo de forma segregada por edificio, facultad, centro o campus, ya que en este estudio se llevó a cabo de forma totalizada. De esta manera, se obtendrían datos más precisos sobre los consumos reales del recurso hídrico en las diferentes áreas de la institución y, dependiendo de los resultados, se podrían aplicar medidas ambientales necesarias.

\section{Conclusiones}

La huella hídrica azul institucional de la UNA expresó una disminución de este indicador durante el periodo de estudio, pasando de 966432 metros cúbicos en el año 2012 a 877062 metros cúbicos en el 2013; posteriormente, una huella hídrica azul de 
895204 metros cúbicos en el 2014, de 888 693 metros cúbicos en el 2015 y, al final, de 891976 metros cúbicos en el 2016. Entre el año 2012 al 2016 se presentó una reducción del ocho por ciento. El resultado se debe principalmente a la gran disminución en el consumo de agua que se ha venido fomentando en los últimos años y que compensó el aumento de la demanda total de energía eléctrica al año.

El agua virtual, que es producto del consumo de energía eléctrica, es el componente con un porcentaje mayor en el cálculo del indicador. Del Gráfico 2 se concluye que la participación de la energía eléctrica en el cálculo de la huella hídrica azul es de suma importancia, ya que representa dos terceras partes de la totalidad; mientras que los otros tres factores han presentado comportamientos a la baja y en conjunto representan un peso relativo menor al $50 \%$ en el total de la huella hídrica institucional.

Es importante indicar que la huella hídrica tiene como principal fin determinar si hay un exceso del consumo del recurso hídrico y corregir esta situación buscando siempre un consumo ambientalmente responsable y sustentable para las demandas futuras, para esto se implementan medidas ambientales que, en el caso de este estudio, demuestran resultados positivos con una disminución durante el periodo de estudio.

En cuanto al cálculo de la huella hídrica azul a nivel per cápita se pudo determinar que se ha venido disminuyendo, y en total se rebajó en un $25 \%$ entre el 2012 y el 2016, indicador positivo para medir las acciones y medidas implementadas en pro de la sustentabilidad ambiental. Es importante mencionar que en este indicador también incidió el incremento anual de la población universitaria durante el periodo de estudio. Se aumentó un cuatro por ciento $(4 \%)$ en el año 2013 con respecto al 2012, posteriormente aumentó un quince por ciento $(15 \%)$ en el 2014, un once por ciento (11\%) en el $2015 \mathrm{y}$, por último, disminuyó un dos por ciento (2\%) en el 2016. Esto representó un aumento de la población de un $31 \%$ en el periodo de estudio.

Se debe continuar con la medición anual del indicador de huella hídrica, con el fin de poder implementar acciones estratégicas que integren la colaboración y participación activa de la toda la comunidad universitaria para mitigar, minimizar o compensar el impacto ambiental que puede ocurrir en la institución, debido a su quehacer diario. Sin embargo, se deben incrementar los esfuerzos para afrontar los continuos retos ambientales orientando a la institución hacia un desarrollo sustentable con un compromiso integral de funcionarios y estudiantes.

\section{Declaración}

Declaramos que no tenemos ningún tipo de interés particular en favorecer o poner en desventaja alguna de las marcas mencionadas tras el análisis. Además, no recibimos apoyo económico que pueda ocasionar algún conflicto ético en los resultados del estudio.

\section{Referencias}

Allan, T. (1993). Priorities for water resources allocation and management. London, Inglaterra: Overseas Development Administration. Recuperado de https:/www.ircwash.org/sites/ default/files/210-93PR-11967.pdf

Agudelo, R. M. (2005). El agua, recurso estratégico del siglo XXI. Revista Facultad Nacional de Salud Pública, 23(1), 91-102. Recuperado de http://aprendeenlinea.udea.edu.co/revistas/ index.php/fnsp/article/view/522

Castillo, M. (2016). Huella hídrica del campus de la Pontificia Universidad Católica del Perú en el 2014 (Tesis de Licenciatura). 
Universidad Católica del Perú, Perú. Recuperado de http://tesis.pucp.edu.pe/repositorio/ handle/20.500.12404/7633

Chavarría, F., Garita, N. y Gamboa, R. (2015). Indicadores de gestión ambiental: Instrumento para medir la calidad ambiental de la Universidad Nacional de Costa Rica. Revista de Ciencias Ambientales, 49(1), 37-54. Recuperado de https://www.revistas.una.ac.cr/index. php/ambientales/article/view/7692/8278

Chavarría, F. (2019). Control ambiental institucional. El caso de la Universidad Nacional Costa Rica. V Congreso Iberoamericano sobre Ambiente y Sustentabilidad en la Universidad Estatal del Sur de Manabí, Ecuador, 5 (1), 87-101.

Contreras, Y., \& Torres, C. M. (2016). Cuantificación de la huella hídrica en las instalaciones de la Universidad de Córdoba Campus Montería, para el año 2014 (Tesis de Licenciatura). Universidad de Córdoba, Colombia. Recuperado de http://repositorio.unicordoba. edu.co/handle/ucordoba/471

Fundación MAPFRE. (2011). Huella hídrica, desarrollo y sostenibilidad en España. Madrid, España: FUNDACIÓN MAPFRE. Recuperado de http://www.comunidadism.es/wp-content/ uploads/downloads/2013/03/informe-huella-hidrica-y-desarrollo-sostenible.pdf

Gerbens-Leenes, P., Hoekstra, A. \& Van der Meer, Th. (2009). The water footprint of energy from biomass: a quantitative assessment and consequences of an increasing share of bio-energy in energy supply. Ecological Economics, 68(4), 1052-1060. Recuperado de https://waterfootprint.org/media/downloads/ Gerbens-Hoekstra-VanderMeer-2008-waterfootprint-bioenergy.pdf

Hoekstra, A. (2003). Virtual water trade: proceedings of the International Expert Meeting on Virtual Water Trade. Netherlands: IHE Delft. Recuperado de http://www.waterfootprint. org/Reports/Report12.pdf.

Hoeskstra, A. \& Chapagain, A. (2004). Water footprints of nations. UNESCO-IHE.
Hoekstra, A. (2008). Water Neutral: reducing and offsetting the impacts of water footprints. Netherlands: UNESCO-IHE. Recuperado de http://citeseerx.ist.psu.edu/viewdoc/download? doi=10.1.1.593.2649\&rep=rep $1 \&$ type $=$ pdf

Hoekstra, A. Chapagain, A. Aldaya, M. y Mekonnen, M. (2011). The Water Footprint Assessment Manual: setting the global standar. London, Inglattera, Washington, DC, Estados Unidos: Earthscan. Recuperado de https://waterfootprint.org/media/downloads/TheWaterFootprintAssessmentManual 2.pdf

Loaiza, P. M. \& Quiceno, A. C. (2018). Cálculo de la huella hídrica en universidades, caso de estudio Universidad Católica de Manizales, en el marco del Sistema de Gestión Ambiental (Tesis de Licenciatura). Universidad Católica de Manizales, Colombia. Recuperado de http://repositorio.ucm.edu.co:8080/jspui/ bitstream/handle/10839/2362/Pamela\%20 Mar\%C3\%ADa\%20Loaiza\%20G\%C3\%B3 mez.pdf? sequence $=1 \&$ isAllowed $=y$

Universidad Nacional, Costa Rica. (2017). Plan del Programa de Gestión Ambiental Institucional de la Universidad Nacional de Costa Rica PGAI-UNA 2017-2021. Heredia, Costa Rica.

Van Oel, P. \& Hoekstra, A. (2010). The green and blue water footprint of paper products: Methodological considerations and quantification. Netherlands: UNESCO-IHE Institute for Water Education. Recuperado de https:// ris.utwente.nl/ws/portalfiles/portal/5146588/ IHE-46_2010.pdf

Vázquez, R. y Buenfil, M. (2012). Huella hídrica de América Latina: Retos y oportunidades. Aqua-LAC, 4(1), 41 - 48. Recuperado de http://www.huellahidrica.org/Reports/Vazquez $\% 20$ del $\% 20$ Mercado $\% 20$ Arribas $\% 20$ and\%20Buenfil\%20(2012).pdf

Water Footprint Network. (2016). Product Water Footprints [Mensaje en un blog]. Recuperado de https://waterfootprint.org/en/resources/ interactive-tools/product-gallery/.

\section{(C) $(1) \Theta \Theta$}

Medición de la huella hídrica de la Universidad Nacional en Costa Rica del año 2012 al 2016 (Fabián Chavarría-Solera y otros) in Uniciencia is protected by Attribution-NonCommercial-NoDerivs 3.0 Unported (CC BY-NC-ND 3.0) 